\title{
Origin of the surface metallization in single-domain $\mathrm{K} / \mathrm{Si}(100) 2 \times 1$
}

\author{
P. Segovia, G. R. Castro, A. Mascaraque, P. Prieto, H. J. Kim,* and E. G. Michel \\ Departamento de Física de la Materia Condensada and Instituto Universitario de Ciencia de Materiales "Nicolás Cabrera," \\ Universidad Autónoma de Madrid, 28049 Madrid, Spain \\ (Received 12 July 1996; revised manuscript received 23 August 1996)
}

\begin{abstract}
The electronic structure and the metallization onset of single-domain $\mathrm{K} / \mathrm{Si}(100) 2 \times 1$ have been investigated with angle-resolved polarization-sensitive ultraviolet photoemission. The electronic states producing the surface metallization have been studied for increasing $\mathrm{K}$ coverages up to room-temperature saturation. As $\mathrm{K}$ coverage increases, the interface undergoes a transition at a critical coverage, from a low-coverage semiconducting phase, to a saturation-coverage metallic phase. Two different surface states $\left(F_{1}\right.$ and $\left.F_{2}\right)$ have been detected in the vicinity of the Fermi level. These two states are sequentially filled along the metallization process. The coverage dependence of both $F_{1}$ and $F_{2}$, and their symmetry properties indicate that the metallization is due to the filling of an initially empty surface band (appearance of $F_{2}$ ). We relate $F_{1}$ to the completion of $\mathrm{K}$ chains in the single-domain surface. The changes detected in $\mathrm{K} 3 p$ line shape correlate well with the modifications of the valence band, and support that the surface remains semiconducting up to the filling of $F_{2}$. [S0163-1829(96)51844-8]
\end{abstract}

The investigation of the electronic and structural properties of $\mathrm{K} / \mathrm{Si}(100) 2 \times 1$ has received widespread attention, ${ }^{1}$ but there exists still controversy about the electronic nature of the interface as a function of $\mathrm{K}$ coverage. Both a metallic ${ }^{2-4}$ and a semiconducting ${ }^{5}$ interface have been found at room temperature (RT, $300 \mathrm{~K})$ saturation coverage. We refer the reader to Refs. $1-8$ for an overview on our current understanding of $\mathrm{K} / \mathrm{Si}(100) 2 \times 1$. Several reasons explain the difficulties found, as the high reactivity of the surface, or the fact that the desorption temperature of bulk $\mathrm{K}$ adsorbed on $\mathrm{Si}$ is close to RT. ${ }^{3,9}$ Both facts make it difficult to compare results from different groups, unless special care is taken. Also, the geometric arrangement of $\mathrm{K}$ atoms at the surface may depend strongly not only on the $\mathrm{K}$ coverage, but also on the surface preparation conditions. ${ }^{6,7}$

We set out in this work to characterize in detail the electronic states giving rise to the surface metallization. These states appear as small peaks close to the Fermi energy $\left(E_{F}\right)$ in the coverage range close to RT saturation. The characterization stresses their symmetry properties and their dependence with $\mathrm{K}$ coverage. This information is crucial to understand their origin and the process of surface metallization.

The experiments have been carried out in an ultrahigh vacuum (UHV) chamber permanently located at the exit of a SEYA 1-m monochromator receiving synchrotron light from the DORIS III storage ring of the Hamburger Synchrotronstrahlungslabor (HASYLAB). It was equipped with facilities for low-energy electron diffraction (LEED), Auger electron spectroscopy (AES), and angle-resolved ultraviolet-photoemission spectroscopy (ARUPS). The position of $E_{F}$ was obtained from reference measurements on a Ta foil in electric contact with the sample end. The work function $\Phi$ was determined from the low-energy cutoff of the secondary electrons. $n$-doped $\mathrm{Si}(100)$ samples were intentionally miscut $4^{\circ}$ towards the [011] direction, and cleaned in UHV by annealing at $1200{ }^{\circ} \mathrm{C}$. For this particular miscut angle, the terraces are separated by double-height steps. ${ }^{10,11}$ Accordingly, sharp single-domain $2 \times 1$ LEED patterns were regularly ob- tained, without traces of a second domain. $\mathrm{K}$ was evaporated from a commercial SAES-Getters source throughly degassed. The base pressure of the system was $3 \times 10^{-11} \mathrm{mbar}$, and during evaporation never rose above $6 \times 10^{-11}$ mbar. Even under these vacuum conditions the sample life before detection of contaminants was limited to $\sim 4-5$ hours. The $\mathrm{K}$ coverage was determined from both the K-induced workfunction change and from the $\mathrm{K} 3 p$ intensity evolution. ${ }^{3} \mathrm{We}$ define in this article $1 \mathrm{ML}$ coverage of potassium $\left(\Theta_{\mathrm{K}}\right)$ as an amount of $\mathrm{K}$ atoms equal to the atomic density of the $\mathrm{Si}(100)$ surface (i.e., $1 \mathrm{ML}=6.78 \times 10^{14}$ atoms $/ \mathrm{cm}^{2}$ ).

The band structure of the clean single-domain $\operatorname{Si}(100) 2 \times 1$ surface was measured in order to characterize the substrate. The results do not differ much from those in Refs. 12 and 13. The top spectrum in Fig. 1 (lower panel) corresponds to the $\bar{\Gamma}_{0}$ point of the clean surface. The peak at $0.7 \mathrm{eV}$ binding energy (BE) is the dangling-bond surface state. ${ }^{12,13}$ No other emission up to $E_{F}$ is detected. In particular, we note that some authors have found peaks in this area which were attributed either to defect states of the clean surface, ${ }^{14}$ or to population of an empty band due to a high sample doping. ${ }^{15}$

The upper panel of Fig. 1 presents the evolution of the $\mathrm{K}$-induced work-function change as a function of $\mathrm{K}$ evaporation time at RT. In agreement with previous results, the work-function decrease reaches a final value of $\Delta \Phi=-3.3$ $\mathrm{eV}$. $\mathrm{K}$ deposition induces an overall shift of the valence band towards higher BE. ${ }^{3}$ The intensity of $\mathrm{K} 3 p$ was also monitored during the deposition (upper panel of Fig. 1). It grows linearly with evaporation time, until it saturates at a mean value of $\sim 6.5 \mathrm{~min}$, as $\Delta \Phi$ does. We will refer to this common RT saturation coverage as $\Theta_{\text {sat }}$.

From $\Theta_{\mathrm{K}}=0.75 \Theta_{\text {sat }}$, a peak is observed at $0.1 \mathrm{eV} \mathrm{BE}$ at $\bar{\Gamma}_{0}$. We will label this peak as $F_{2}$. It does not disperse with the photon energy (see Fig. 2, left panel), although its intensity changes strongly, showing a maximum at $\sim 12 \mathrm{eV}$ photon energy. Its symmetry and dispersion along the Brillouin zone were measured at this energy. $F_{2}$ is observed only for 

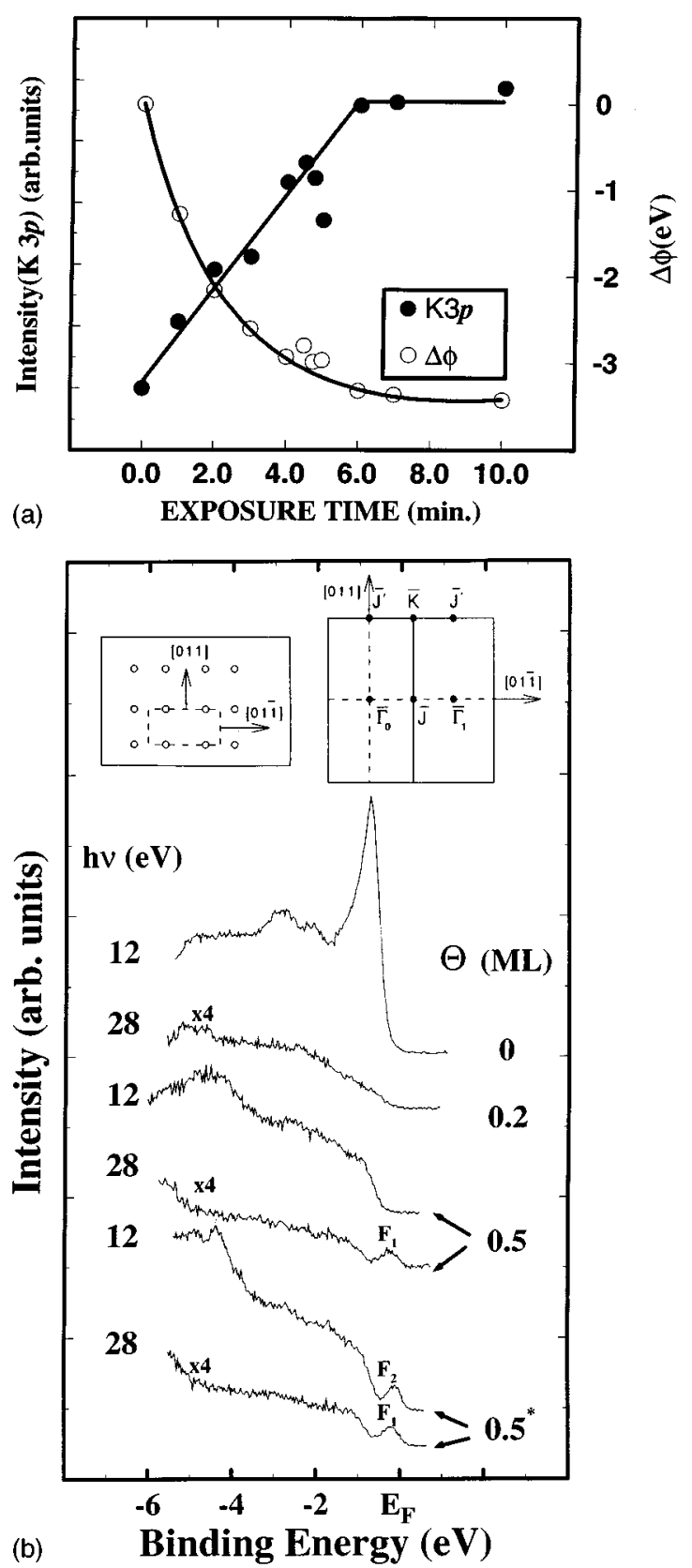

FIG. 1. Lower panel: coverage dependence of $F_{1}$ and $F_{2}$. The spectra are taken at the resonance conditions of $F_{2}(h \nu=12 \mathrm{eV})$ and $F_{1}(h \nu=28 \mathrm{eV})$. The former were taken at $\bar{\Gamma}_{0}$ and the latter at $\bar{\Gamma}_{1}$. A saturation coverage of $0.70 \mathrm{ML}$ was assumed in the absolute coverages cited. The two bottom spectra (coverage $0.5^{*} \mathrm{ML}$ ) were obtained after adding $0.05 \Theta_{\text {sat }}$ to the spectra at $0.5 \mathrm{ML}$. The inset depicts the surface geometry in real and reciprocal space. Upper panel: work-function change (empty circles) and $\mathrm{K} 3 p$ core level intensity (filled circles), as a function of $\mathrm{K}$ evaporation time.

$p$ geometry (incident light grazing to the surface, see Fig. 3), indicating a major $s-p_{z}$ character. As shown in Fig. 3 (upper panel), the tail of $F_{2}$ crosses $E_{F}$, denoting a metallic surface. $F_{2}$ is concentrated in the area close to $\bar{\Gamma}$ points because it disperses and disappears when going towards $\overline{\mathbf{J}}$ or $\overline{\mathbf{J}^{\prime}}$ points. $F_{2}$ is extremely sensitive to minute amounts of surface contamination. Considering these properties (sensitivity to contamination, location in a surface band gap, lack of dispersion

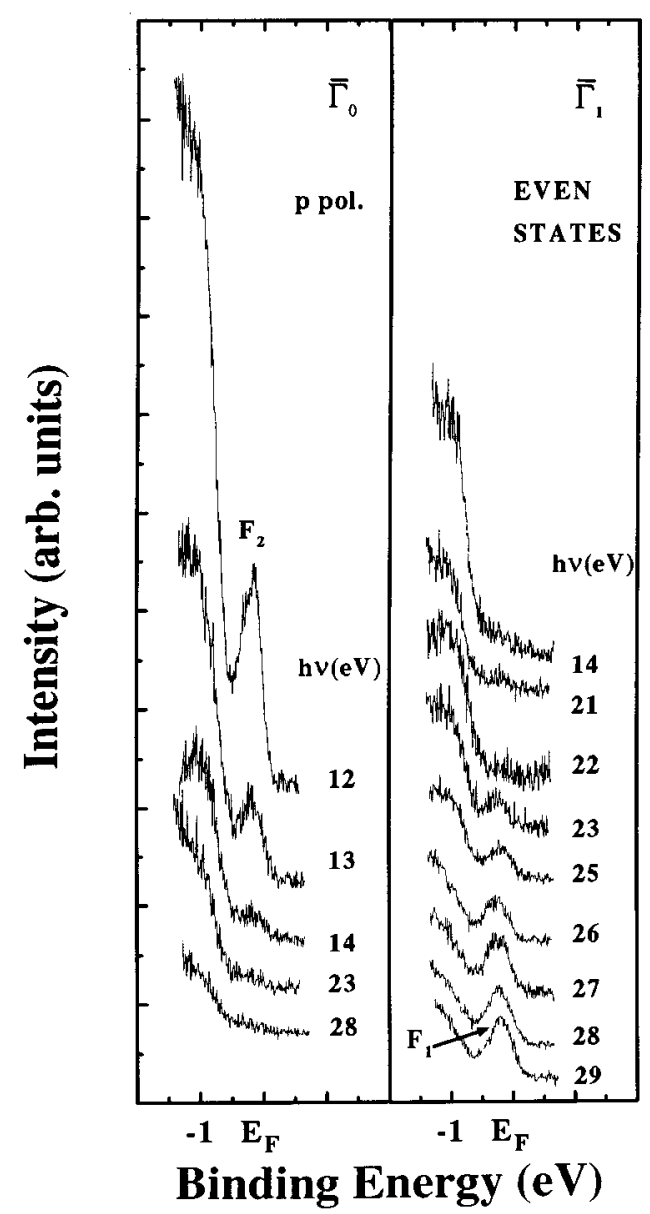

FIG. 2. Photon energy dependence of the surface states $F_{2}$ (left panel) and $F_{1}$ (right panel). The data have been taken at $\bar{\Gamma}_{0}$ for $F_{2}$ ( $p$ polarization), and at $\bar{\Gamma}_{1}$ for $F_{1}$ (even states with respect to $[01 \overline{1}]$ plane). Note the strong resonances at $12 \mathrm{eV}\left(F_{2}\right)$ and $28 \mathrm{eV}$ $\left(F_{1}\right)$, respectively.

with $k_{\perp}$ ), we assign $F_{2}$ to a surface state whose origin will be discussed below.

As the $\mathrm{K}$ coverage is decreased, the main change detected is the disappearance of $F_{2}$ below $\Theta_{\mathrm{K}}=0.70 \Theta_{\text {sat }}$. Thus, at $\Theta_{\mathrm{K}}=0.70 \Theta_{\text {sat }}$ there is no $F_{2}$ emission, but it abruptly appears after adding a coverage of only $0.05 \Theta_{\text {sat }}$ (see Fig. 1). No other peaks close to $E_{F}$ are detected for $\Theta_{\mathrm{K}}<0.70$ $\Theta_{\text {sat }}$. Nevertheless, there is a narrow coverage range around $0.70 \Theta_{\text {sat }}$ (i.e., before the appearance of $F_{2}$ ) where a second peak $F_{1}$ is detected at $0.20 \mathrm{eV} \mathrm{BE}$ (see Figs. 1, 2, and 3). This peak is weaker than $F_{2}$, and has different properties. Its intensity is highest at $\bar{\Gamma}_{1}$ (see Figs. 1 and 3) for $h \nu=28 \mathrm{eV} .{ }^{16}$ It does not disperse with $k_{\perp}$. Two different geometries were used to detect states with even parity with respect to the mirror planes containing the $[011]$ and $[01 \overline{1}]$ directions (see Fig. 1, inset of lower panel). $F_{1}$ contains only even states with respect to the mirror plane along [011] (see Fig. 3).

From the point of view of the metallic or semiconducting character of the interface, we can summarize the results as follows: three different coverage regimes were found. For $\Theta_{\mathrm{K}} \leqslant 0.70 \Theta_{\text {sat }}$ the surface is semiconducting, and the band structure is not much different from that of the clean 


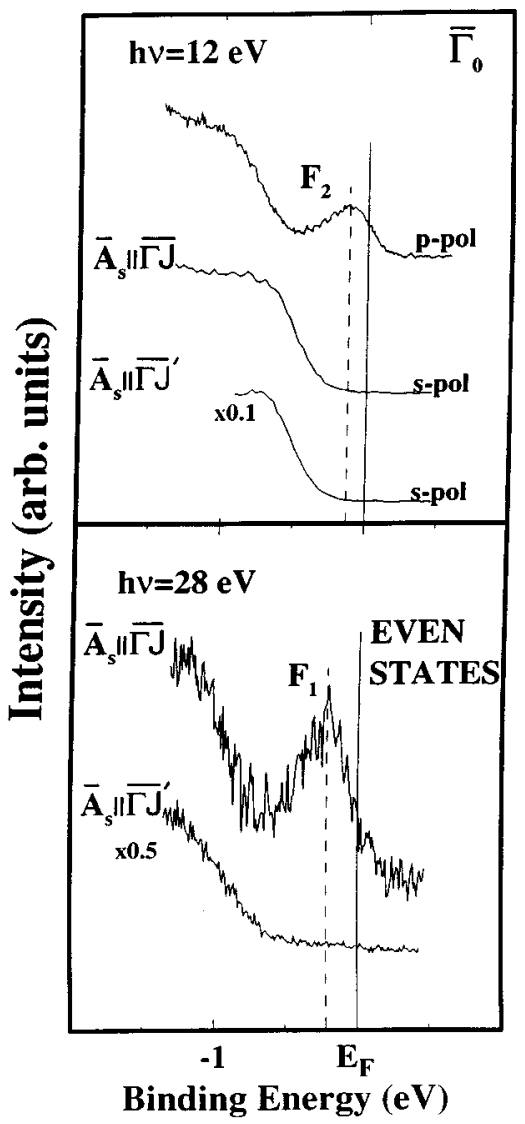

FIG. 3. Symmetry properties of the surface states $F_{2}$ (upper panel) and $F_{1}$ (lower panel). $F_{2}$ is detected only under $p$ polarization. In the lower panel the experimental geometry allows only the detection of even states with respect to the mirror planes [011] and [011], respectively. The top spectrum of lower panel was taken at the first $\bar{\Gamma}$ point along the $[01 \overline{1}]$ direction (i.e., $\bar{\Gamma}_{1}$ in Fig. 1$)$. The bottom spectrum of lower panel was taken at the first $\bar{\Gamma}$ point along the [011] direction. Note the small difference in binding energies between $F_{2}(0.1 \mathrm{eV})$ and $F_{1}(0.2 \mathrm{eV})$ (dashed lines).

$\mathrm{Si}(100) 2 \times 1$ surface (see also below for more details). For $\Theta_{\mathrm{K}} \geqslant \Theta_{\text {sat }}$, a surface band $F_{2}$ of $s-p_{z}$ character crosses $E_{F}$. For a coverage equal to $0.70 \Theta_{\text {sat }}$, a second surface band $F_{1}$ was detected. The metallization evidenced by the observation of $F_{2}$ correlates with changes in the $\mathrm{K} 3 p$ line shape and $\mathrm{BE}$ indicating also the formation of a metallic interface. ${ }^{17}$

We consider now the origin of $F_{2}$. A similar peak has been detected previously, ${ }^{2,4,5,8,18}$ but the lack of a complete characterization hindered a final assignment, and thus different interpretations on its origin were proposed. Enta et al. ${ }^{18}$ and Abukawa et al. ${ }^{5}$ attributed the peak to excess $\mathrm{K}$ atoms above $1 \mathrm{ML}$. This interpretation can be discarded in view of the periodicity and defined symmetry of both $F_{1}$ and $F_{2}$, which would not be expected for randomly distributed excess $\mathrm{K}$ atoms. Chao et al. ${ }^{8}$ related a similar peak with the surface metallization, following Johansson and Reihl, ${ }^{2}$ who found an unoccupied peak moving towards $E_{F}$ as the coverage increased, and crossing $E_{F}$ at a coverage close to the minimum of the work function $(\sim 0.5 \mathrm{ML})$. At this coverage, they detected also an occupied state using unpolarized radiation at $21.2 \mathrm{eV}$. The symmetry properties of $F_{2}$ agree well with the results of Johansson and Reihl for the empty part of the band at lower coverages. Thus, our results show the counterpart of the unoccupied band found in Ref. 2, and support the idea that the surface metallization proceeds by filling of an initially empty surface band. The whole process takes place in a narrow coverage range around $0.75 \Theta_{\text {sat }}$. Ishida and Terakura ${ }^{19}$ have performed first-principles calculations within the local-density-functional theory, while Kobayashi et al. ${ }^{20}$ applied first-principles molecular dynamics based on the norm-conserving pseudopotential to $\mathrm{K} / \mathrm{Si}(100) 2 \times 1$. Their results shed light on the origin of $F_{2}$. The main effect of $\mathrm{K}$ adsorption on cave $\left(T_{3}\right)$ sites ${ }^{19-21}$ is to induce a shift of the two surface states of the clean $\mathrm{Si}(100) 2 \times 1$ surface towards higher BE $\left(S_{1}\right.$ and $S_{2}$ in Ref. 20 ), with partial filling of $S_{2}$. These two bands also become closer in energy after $\mathrm{K}$ adsorption. On the other hand, occupation of pedestal $(H H)$ sites $^{20}$ splits a third surface band ( $S_{3}$ in Ref. 20), located in energy above $S_{1}$ and $S_{2}$, and initially unoccupied. A parabolic upwards dispersion away from $\bar{\Gamma}$ was predicted, both along $\overline{\Gamma \mathrm{J}}$ and $\overline{\Gamma \mathrm{J}^{\prime}}$ directions. The orbital origin of this band was analyzed in an earlier study, ${ }^{19}$ where it was found mainly an $s-p_{z}$ character. We interpret $F_{2}$ as being due to a partial occupation of $S_{3}$ band, since both $S_{3}$ and $F_{2}$ have the same symmetry and comparable dispersions. ${ }^{17}$ In view of the results in Ref. 20, the observation of $F_{2}$ would be indicative of occupation of pedestal $(\mathrm{HH})$ sites, since only when these positions are occupied is an $S_{3}$ band split. Thus, the surface at saturation coverage should comprise areas with two adsorption sites, giving rise to the occupation of $F_{2}$, in agreement with photoelectron diffraction results. ${ }^{22,23}$ At this moment it is interesting to discuss the results obtained for the rest of the band structure. As we mentioned above, we have investigated the evolution of $S_{1}$ and $S_{2}$ bands as a function of K coverage. Our results do not differ much from a previous study. ${ }^{5}$ In both cases the experimental points have been compared with the calculations for $1 \mathrm{ML} \mathrm{K} .{ }^{20}$ In order to get a good matching between the experimental points and calculations, ${ }^{20}$ it was necessary to shift the theoretical bands by $\sim 0.6 \mathrm{eV}$ towards lower binding energies. This value is within reasonable limits, taking into account the difficulties to account for exchange and correlation energies in the calculation. Interestingly, such a shift brings $S_{3}$ below the Fermi level (see also below). A different interpretation was proposed in Ref. 4 where two peaks close to $E_{F}$ (similar to $F_{2}$ and $F_{1}$ ) were attributed to the two surface bands $S_{1}$ and $S_{2}$ themselves. ${ }^{19,20}$ We discard this possibility in view of the excellent agreement with theory found when $S_{1}$ and $S_{2}$ are attributed to higher $\mathrm{BE}$ bands. ${ }^{5}$

In the coverage range close to $0.70 \Theta_{\text {sat }}$, only the second surface state $F_{1}$ was observed, with a surface still semiconducting (see Fig. 3). No states with even parity with respect to the [011] plane were found. This asymmetric behavior suggests a differential strength of the adatom-adatom inter-

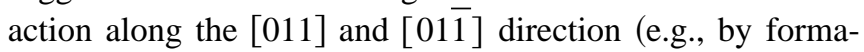
tion of well-ordered atomic chains along the [011] direction). An enhanced interaction along the chains could result in the formation of an orbital of $\pi$-like symmetry in the plane of the surface. The complete characterization of this orbital re- 
quires detection of electronic states with odd symmetry with respect to the mirror planes. Work is in progress to clarify this point. We can only speculate on the origin of $F_{1}$, but an appealing possibility is that its appearance is related to the order-disorder transition observed at a coverage close to $\Theta_{\text {sat. }}{ }^{6}$

An important question is the precise coverage at which the metallization takes place. In view of the sequential filling of $F_{1}$ and $F_{2}$ (the latter producing a metallic surface), the process is probably related to occupation of a second site. As the coverage is decreased, the amount of $\mathrm{K}$ at the second site should diminish accordingly. This is expected for an interface in equilibrium due to the difference in bonding energies between both sites. ${ }^{3,9}$ Nevertheless, the difference is very small. It is thus possible to produce metastable surfaces with coverages below $\Theta_{\text {sat }}$, and two coexisting sites. ${ }^{3}$ Several techniques have shown that the mean distributions of $\mathrm{K}$ atoms on the surface depends very much on the preparation conditions. ${ }^{3,6}$ Although cave sites are always favored over pedestal sites, under some preparation conditions a comparable population of each type of site can be found. In this case, the onset for $S_{3}$ observation could vary, depending on the degree of surface ordering, explaining the differences found in the literature. This result has been evidenced by scanning tunneling microscopy work, and could be enhanced by inadequate surface cleanliness. ${ }^{24}$ In our case, once the coverage decreases down to $0.70 \Theta_{\text {sat }}, F_{2}$ disappears rapidly. An absolute coverage calibration, which would fix the metallization onset, is difficult on the basis of our data alone. Previous results with quantitative techniques sensitive to the amount of $\mathrm{K}$ at the surface have found RT saturation coverages of 0.6-0.7 ML (Ref. 3) or 0.98 $\pm 0.05 \mathrm{ML}^{25}$ The main source of error for these numbers comes from inaccuracies in the actual surface temperature during evaporation, which can change the saturation value significantly. ${ }^{9}$ If $\Theta_{\text {sat }}$ varies within these two limits, the Fermi level onset detected here at $0.73 \Theta_{\text {sat }}$ would correspond to an absolute coverage between 0.5 and $0.7 \mathrm{ML}$. This conclusion agrees well with other experimental evidences supporting a metallization onset above $\mathrm{e}^{3,6,26-28} 0.5 \mathrm{ML}$ and with the existence of two adsorption sites at $\Theta_{\text {sat }}{ }^{22,23}$ The overall band topology found in the experiment is reasonably well described by the theory, ${ }^{20}$ but this is not the case for the metallization onset, where there are important discrepancies. From the experimental point of view the process of metallization is as follows: the surface is semiconducting up to a critical coverage $(\sim 0.5-0.7 \mathrm{ML})$, and it remains metallic for higher coverages up to multilayers. ${ }^{3,28}$ This behavior is in contradiction with the evolution expected within the framework of one-electron band theory, since the surface should metallize at some point below $0.5 \mathrm{ML}$, to become semiconducting at $1 \mathrm{ML}$, and then again metallic. ${ }^{1,29}$ Correlation effects have been considered to explain the lack of metallicity below $0.5 \mathrm{ML},{ }^{1}$ and a possible charge trapping has been recently invoked to explain the metallic character at $1 \mathrm{ML},{ }^{29}$ but a complete explanation of the whole process described in this article is still lacking. ${ }^{29}$

In conclusion, we have found that the RT saturated $\mathrm{K} / \mathrm{Si}(100) 2 \times 1$ surface is metallic. The metallization takes place in a narrow coverage range by filling of an initially empty surface band of $s-p_{z}$ character. Before metallization, another surface peak $F_{1}$ was found. Its symmetry properties suggest an origin related to the formation of well ordered $\mathrm{K}$ chains at the surface.

This work was financed by DGICYT (Spain) under Grants Nos. PB-94-1527 and SAB-950019P.
*Present address: II Institut für Experimentalphysik, Luruper Chaussee 149, 22761 Hamburg, Germany.

${ }^{1}$ For a recent review, see F. Flores, Surf. Rev. Lett. 2, 513 (1995).

${ }^{2}$ L.S.O. Johansson and B. Reihl, Phys. Rev. Lett. 67, 2191 (1991).

${ }^{3}$ E.G. Michel et al., Phys. Rev. B 45, 11811 (1992).

${ }^{4}$ J.A. Martin-Gago et al., Surf. Sci. 307-309, 995 (1994).

${ }^{5}$ T. Abukawa et al., Surf. Sci. 261, 217 (1992).

${ }^{6}$ P. Soukiassian et al., Phys. Rev. 46, 13471 (1992).

${ }^{7}$ U.A. Effner et al., Surf. Sci. 277, 207 (1992).

${ }^{8}$ Y.-C. Chao et al., Phys. Rev. B 52, 2579 (1995).

${ }^{9}$ S. Tanaka et al., Phys. Rev. B 42, 1868 (1990).

${ }^{10}$ R. Kaplan, Surf. Sci. 93, 145 (1980).

${ }^{11}$ O.L. Alerhand et al., Phys. Rev. Lett. 64, 2406 (1990).

${ }^{12}$ L.S.O. Johansson et al., Phys. Rev. B 42, 1305 (1990).

${ }^{13}$ Y. Enta et al., J. Phys. Soc. Jpn. 59, 657 (1990).

${ }^{14}$ A. Goldmann et al., Surf. Sci. 169, 438 (1986).

${ }^{15}$ P. Mårtensson, A. Cricenti, and G.V. Hansson, Phys. Rev. B 33, 8855 (1986).

${ }^{16}$ This behavior is due to the fact that the projected bulk bands at $\bar{\Gamma}_{0}$ and $\bar{\Gamma}_{1}$ (see Fig. 1) are not equivalent. A more detailed analy- sis will be presented in a forthcoming publication (Ref. 17).

${ }^{17} \mathrm{P}$. Segovia et al. (unpublished).

${ }^{18}$ Y. Enta et al., Phys. Rev. B 39, 1125 (1989).

${ }^{19}$ H. Ishida and K. Terakura, Phys. Rev. B 40, 11519 (1989).

${ }^{20}$ K. Kobayashi et al., Phys. Rev. B 45, 3469 (1992).

${ }^{21}$ Most recent investigations support initial adsorption on cave sites only, or on a mixture of sites including cave sites.

${ }^{22}$ T. Abukawa and S. Kono, Phys. Rev. B 37, 9097 (1988).

${ }^{23}$ M.C. Asensio et al. (unpublished).

${ }^{24}$ Soukiassian et al. (Ref. 6) have pointed out that minute amounts of contaminants can also increase the saturation value, probably by forming less volatile $\mathrm{K}$ compounds.

${ }^{25}$ A.J. Smith, W.R. Graham, and E.W. Plummer, Surf. Sci. Lett. 243, L37 (1991)

${ }^{26}$ A. Brodde, Th. Bertrans, and H. Neddermeyer, Phys. Rev. B 47, 4508 (1993)

${ }^{27}$ G.R. Castro et al., Vacuum 41, 564 (1990).

${ }^{28}$ A. Hamawi, Phys. Rev. B 50, 10910 (1994).

${ }^{29}$ M.C. Refolio et al., Phys. Rev. B 53, 4791 (1995). 